\title{
Usefulness of Prostate-Specific Antigen Density as an Indicator for Recommending Prebiopsy Magnetic Resonance Imaging to Prevent Missed Prostate Cancer Diagnoses
}

\author{
Jin Hyung Jeon, Kyo Chul Koo, Byung Ha Chung, Kwang Suk Lee \\ Department of Urology, Yonsei University College of Medicine, Seoul, Korea
}

Purpose: To identify the indication for recommending prebiopsy magnetic resonance imaging (MRI) to prevent prostate cancer missed diagnoses in cases without prebiopsy MRI.

Materials and Methods: Between January 2017 and September 2020, 585 patients suspected with prostate cancer underwent prostate biopsy after MRI. For patients with visible lesions, MRItargeted biopsy using an image-based fusion program was performed in addition to the 12core systematic biopsy. Patients for whom MRI was performed in other institutions $(n=4)$ and patients who underwent target biopsy alone $(n=7)$ were excluded.

Results: Of 574 patients (median prostate-specific antigen [PSA] level, $6.88 \mathrm{ng} / \mathrm{mL}$; mean age, 68.2 years), 342 (59.6\%) were diagnosed with prostate cancer (visible lesions=312/449 [69.5\%]; nonvisible lesions=30/123 [24.0\%]). The detection rates of visible lesions stratified using the Prostate Imaging Reporting and Data System score (3 vs. 4 vs. 5) were $30.9 \%$ (54 of 175), $61.2 \%$ (150 of 245), and $90.1 \%$ (127 of 141), respectively. Multivariate analysis showed that PSA density was a significant factor for presence of visible lesions, prostate cancer, and significant prostate cancer diagnosis. Among patients with positive lesions, 27 (8.2\%) were diagnosed with prostate cancer concomitant with negative systematic biopsy results. A PSA density of $0.15 \mathrm{ng} / \mathrm{mL} / \mathrm{cm}^{3}$ was identified as the significant cutoff value for predicting positive target biopsy in groups with negative systematic biopsy. Sixty of the negative target lesions (26.1\%) were diagnosed using systematic biopsy.

Conclusions: To maximize cancer detection rates, both targeted and systematic biopsies should be implemented. PSA density was identified as a useful factor for recommending prebiopsy MRI to patients suspected with prostate cancer.

Key Words: Magnetic resonance imaging, Prostate cancer, Prostate-specific antigen
Received May 3, 2021

Revised May 27, 2021

Accepted May 30, 2021

Corresponding author:

Kwang Suk Lee

Email: winner0428@gmail.com https://orcid.org/0000-0002-7961-8393

This research was supported by a grant of Patient-Centered Clinical Research Coordinating Center funded by the Ministry of Health \& Welfare, Republic of Korea (grant number: HI19C0481, HC19C0164).

\section{INTRODUCTION}

Magnetic resonance imaging (MRI) before biopsy is recommended for patients suspected with prostate cancer, according to the National Comprehensive Cancer Network guideline. ${ }^{1}$ There are several known reasons for the efficacy of MRI in improving cancer detection rates. Specifically, for cancers missed at the initial prostate biopsy because of their location in the anterior region of the prostate, MRI improves the prostate cancer diagnostic accuracy as well as enhances the sampling of clinically significant prostate cancer $(\mathrm{csPCa}){ }^{2-4}$ 
Numerous studies related to MRI-targeted biopsy have focused on cancer detection rates and clinical variables. A recent multicenter paired validating confirmatory study reported that MRI is useful for avoiding $27 \%$ of primary biopsies and reducing $5 \%$ of insignificant prostate cancer diagnoses. ${ }^{5}$ To avoid unnecessary prostate biopsy, several studies presented MRI findings and prostate-specific antigen (PSA) density as criteria for decisionmaking. ${ }^{6}$

Nevertheless, the indication for performing expensive MRI for patients suspected with prostate cancer needs to be identified. ${ }^{7}$ To further increase the cost-effectiveness of MRI, an indication for preventing missed prostate cancer diagnoses in cases without prebiopsy MRI is required. Therefore, this study aimed to investigate the factors useful for detecting the presence of visible lesions, predicting cancer diagnosis, and predicting csPCa. Accordingly, we identified the indication for recommending MRI to prevent missed diagnoses in cases of prostate biopsy without MRI.

\section{MATERIALS AND METHODS}

\section{Ethics Statement}

Institutional Review Board approval was obtained for this study to collect data on all patients suspected with prostate cancer at Yonsei University College of Medicine (approval number: 3-20170324). The requirement of written informed consent was waived owing to the retrospective nature of the study.

\section{Study Population and Data Collection}

We reviewed the data of 585 patients suspected with prostate cancer who underwent prostate biopsy after MRI between January 2017 and
September 2020. Among them, patients for whom MRI was performed in other institutions $(n=4)$ and patients who underwent target biopsy alone because of using antiplatelet agents or old age ( $\geq 85$ years) $(n=7)$ were excluded from the analysis; thus, 574 patients were included in the final analysis. Clinical variables relevant to the study included age, serum PSA level, prostate volume, clinical stage, biopsy Gleason score, and MRI findings.

\section{MRI Protocol}

The imaging investigation was performed using a 3.0 Tesla MRI system (Intera Achieva 3.0 T, Phillips Medical System, Best, Netherlands) equipped with a phased array coil ( 6 channels). The prostate MRI protocol involved diffusion-weighted imaging in addition to T2-weighted imaging. T2-weighted turbo spin-echo MRI was acquired in 3 planes (axial, sagittal, and coronal). MRI datasets were obtained at identical slice locations, with a slice thickness of $3 \mathrm{~mm}$ and no intersection gap. Two b-values $(0-1,400)$ were used, and diffusion restriction was quantified via apparent diffusion coefficient (ADC) mapping. Dynamic contrastenhanced MRI was also performed.

Uroradiologists denoted regions of interest for suggested prostate cancer on the ADC maps examined using a Digital Imaging and Communications in Medicine workstation. The Prostate Imaging Reporting and Data System (PI-RADS) version 2 scoring system was used to describe the MRI findings. ${ }^{8}$ The presence of visible lesions was defined as lesions with a PI-RADS score $\geq 3$.

\section{Prostate Biopsy Protocol}

All patients underwent a prostate biopsy procedure performed by a urologist (LKS). One hour after an intravenous injection of third generation 
cephalosporin as a prophylactic antibiotic, all patients underwent prostate biopsy in the left lateral decubitus position. After povidone iodine rectal preparation, all patients received $10 \mathrm{~cm}^{3}$ of $2 \%$ intrarectal lidocaine gel (Instillagel, FARCOPHARMA, Köln, Germany). After 5 minutes, a transrectal probe was inserted and the prostate volume was measured. Then, local anesthesia was induced.

In patients with visible lesions on MRI, 4 MRItargeted cores per target were initially performed. The targets were then obtained, and a 12-core biopsy was performed. MRI-targeted biopsy was performed with an MRI/transrectal ultrasoundfusion-targeted biopsy protocol using the BK3000 ultrasound system embedded side-fire method (BK Medical, Peabody, MA, USA) and an image-based fusion program (BioJet, D\&K Technologies GmbH, Barum, Germany). In patients with nonvisible lesions, only a 12-core biopsy was performed.

\section{Risk Factor Evaluation}

Confounding factors among PSA levels, prostate volume, and PSA density were evaluated. The receiver operating characteristic (ROC) curve analysis for predicting the presence of visible lesions showed that the area under the ROC curve (AUC) values of PSA levels, prostate volume, and PSA density were $0.595(0.542-0.648 ; \mathrm{p}=0.0005)$, $0.574(0.517-0.630 ; \mathrm{p}=0.0108)$, and 0.639 (0.590$0.689, \mathrm{p}<0.0001)$, respectively. For predicting cancer diagnosis and csPCa, the AUC values of PSA density (0.733 [0.692-0.773], p<0.0001; 0.743 [0.702-0.783], $\mathrm{p}<0.0001)$ were significantly higher than those of PSA levels (0.627 [0.582-0.673], $\mathrm{p}=0.0001 ; 0.664[0.620-0.709], \mathrm{p}=0.0001)$ and prostate volume (0.676 [0.632-0.720], $\mathrm{p}<0.0001$; 0.647 [0.602-0.692], $\mathrm{p}<0.0001)$. Therefore, PSA level and prostate volume data were excluded from the multivariate analysis to avoid confounding (Fig. 1).

\section{Statistical Analyses}

Categorical variables were evaluated using the chi-square test and Fisher exact test. Differences in variables with a continuous distribution across categories were assessed using the Mann-Whitney $\mathrm{U}$-test. Multivariate regression analyses were performed on the factors predicting visible lesion, cancer diagnosis, and csPCa (defined as a Gleason score sum $\geq 7$ [3+4]) among target lesions that had a $\mathrm{p}$-value $<0.05$ in the univariate analyses. ROC curves and AUCs were used to obtain the cutoff value. These optimal cutoff values were based on predefined values based on a sensitivity analysis using Youden index (sensitivity+specificity -1$){ }^{9}$ The AUC was compared using the Delong method for statistical significance of differences in AUC. All reported p-values are 2-sided, and statistical significance was set at $<0.05$ Statistical analyses were performed using IBM SPSS Statistics ver. 22.0 (IBM Co., Armonk, NY, USA) and SAS ver. 9.3 (SAS Inc., Cary, NC, USA).

\section{RESULTS}

\section{Demographic Data}

The baseline clinical and demographic characteristics of the patients who underwent prostate biopsy are shown in Table 1. A total of 342 patients (59.6\%) were diagnosed with prostate cancer (visible lesions=312 of 449 [69.5\%]; nonvisible lesions=30 of 123 [24.0\%]). Among them, the proportion of csPCa was $46.7 \%$ (visible lesions $=312$ of 449 [69.5\%]; nonvisible lesions $=30$ of 125 [24.0\%]). The individuals with visible lesions had a significantly higher frequency of prostate cancer and csPCa than those with nonvisible lesions ( $\mathrm{p}<0.0001)$.

Furthermore, the individuals with visible lesions had more advanced age, higher PSA level, PSA density, 

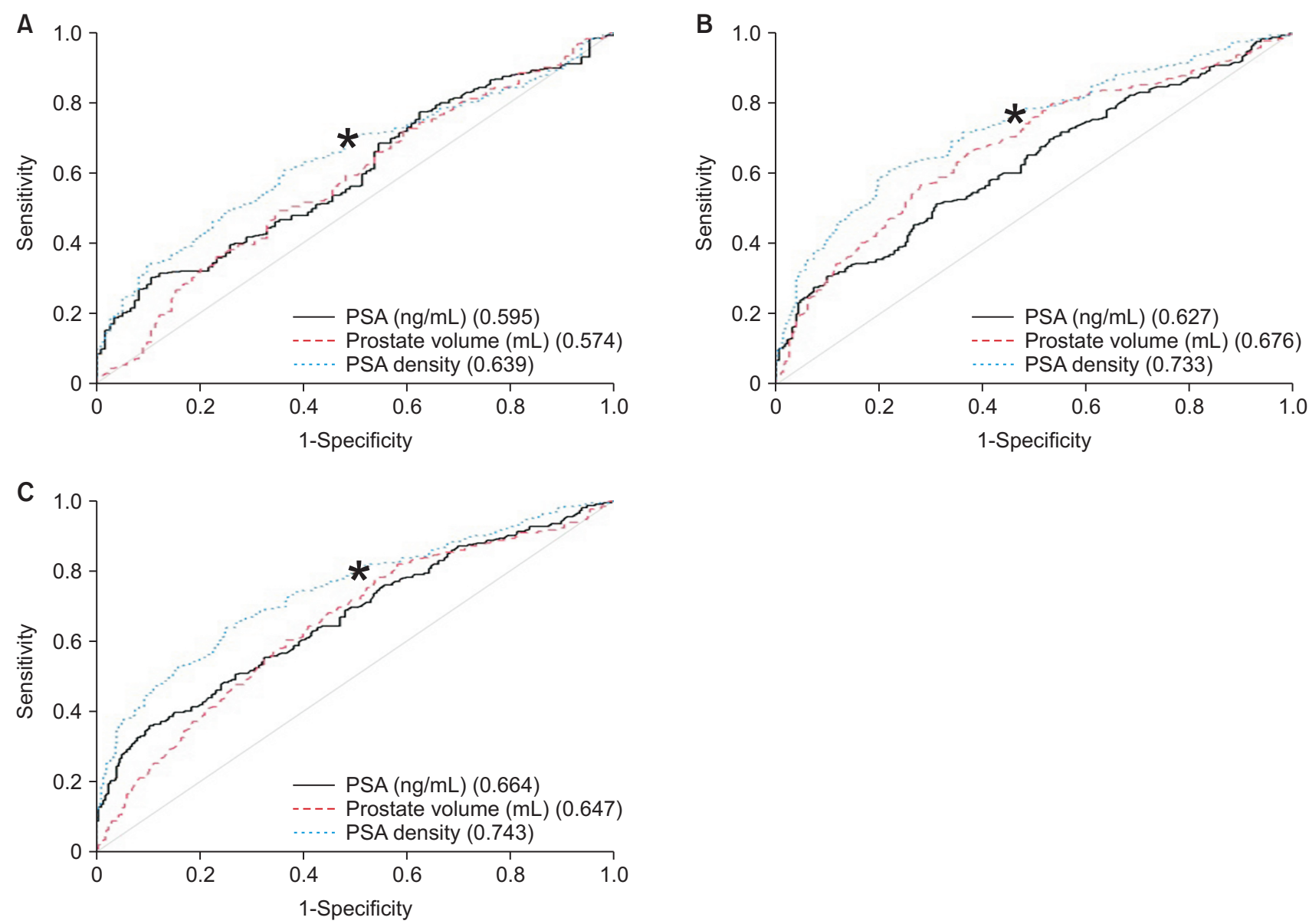

Fig. 1. Receiver operating characteristic curves. Prediction of the presence of visible lesions (A), cancer (B), and significant cancer (C). PSA: prostate-specific antigen. *Indicates the optimal cutoff point which is calculated by Youden index (Sensitivity+Specificity-1).

proportion of previous prostate biopsy history, and lower prostate volume than those with nonvisible lesions ( $\mathrm{n}=449$ vs. 125 ; median age, 69.0 vs. 65.7 years, $\mathrm{p}<0.001$; median PSA levels, $25.07 \mathrm{ng} / \mathrm{mL}$ vs. $6.98 \mathrm{ng} /$ $\mathrm{mL}, \mathrm{p}=0.0002$; PSA density, $0.75 \mathrm{ng} / \mathrm{mL} / \mathrm{cm}^{3}$ vs. 0.18 $\mathrm{ng} / \mathrm{mL} / \mathrm{cm}^{3}, \mathrm{p}=0.0003$; prostate volume, $\mathrm{cm}^{3} 38.0$ vs. $\left.42.1 \mathrm{~cm}^{3}, \mathrm{p}=0.0139\right)$.

The detection rates of visible lesions stratified using the PI-RADS score (3 vs. 4 vs. 5) were $30.9 \%$ (54 of 175), $61.2 \%$ (150 of 245), and $91.1 \%$ (127 of 141), respectively. There is no significant difference in previous prostate biopsy history between the 2 groups (visible lesions $=100$ of 449 [22.3\%]; nonvisible lesions $=29$ of 125 [23.2\%], p=0.826).

\section{Prediction of Cancer Diagnosis and Clinically Significant Prostate Cancer}

Age and PSA density were identified as the significant factors for the presence of visible lesions. Univariate analysis was performed to predict cancer diagnosis and csPCa using several factors, including age, PSA density, and history of prostate biopsy. The multivariate logistic regression analyses identified that age, PSA density, and presence of visible lesions remained as independent predictors of prostate cancer diagnosis and csPCa (Table 2).

The diagnostic performance of PSA density using 
Table 1. Baseline clinical and demographic characteristics of patients suspected with prostate cancer who underwent prostate biopsy after magnetic resonance imaging

\begin{tabular}{|c|c|c|c|}
\hline Characteristic & Visible lesions & Nonvisible lesions & p-value \\
\hline No. of patients & $449(78.2)$ & $125(21.8)$ & \\
\hline Age (yr) & $68.9(60.6-77.2)$ & $65.7(57.2-74.3)$ & 0.0002 \\
\hline PSA level (ng/mL) & $27.9(15.5-40.3)$ & $6.98(2.88-7.01)$ & 0.0004 \\
\hline Prostate volume $(\mathrm{mL})$ & $38.1(20.8-55.3)$ & $42.1(23.8-60.4)$ & 0.0229 \\
\hline PSA density & $0.84(0.43-1.25)$ & $0.18(0.07-0.30)$ & 0.0008 \\
\hline Previous prostate biopsy history & $100(22.3)$ & $29(23.2)$ & 0.8259 \\
\hline Diagnosis of prostate cancer & $312(69.5)$ & $30(24.0)$ & $<0.0001$ \\
\hline Gleason score & & & $<0.0001$ \\
\hline$\leq 6$ & $58(18.6)$ & $16(53.3)$ & \\
\hline$\geq 7(3+4)$ & $254(81.4)$ & $30(24.0)$ & \\
\hline Positive systematic biopsy & $286(63.7)$ & $30(24.0)$ & $<0.0001$ \\
\hline Gleason score & & & 0.0011 \\
\hline$\leq 6$ & $72(25.2)$ & $16(53.3)$ & \\
\hline$\geq 7(3+4)$ & $214(74.8)$ & $30(24.0)$ & \\
\hline Positive target biopsy & $331 / 561(59.0)$ & & \\
\hline PI-RADS 3 & $54 / 175(30.9)$ & & \\
\hline PI-RADS 4 & $150 / 245(61.2)$ & & \\
\hline PI-RADS 5 & $127 / 141(90.1)$ & & \\
\hline
\end{tabular}

Values are presented as number (\%) or median (range).

PSA: prostate-specific antigen, PI-RADS: Prostate Imaging Reporting And Data System.

Table 2. Multivariate analysis of factors predicting cancer and significant prostate cancer

\begin{tabular}{|c|c|c|c|c|}
\hline \multirow{2}{*}{ Variable } & \multicolumn{2}{|c|}{ Univariable analysis } & \multicolumn{2}{|c|}{ Multivariable analysis } \\
\hline & Odds ratio $(95 \% \mathrm{CI})$ & $\mathrm{p}$-value & Odds ratio $(95 \% \mathrm{CI})$ & p-value \\
\hline \multicolumn{5}{|l|}{ Presence of visible lesions } \\
\hline Age (yr) & $1.046(1.022-1.072)$ & 0.0002 & $1.044(1.018-1.070)$ & 0.0007 \\
\hline PSA (ng/mL) & $1.068(1.023-1.115)$ & 0.0025 & - & - \\
\hline Prostate volume $(\mathrm{mL})$ & $0.988(0.977-0.998)$ & 0.0242 & - & - \\
\hline PSA density ( 0.1 unit) & $1.401(1.183-1.660)$ & $<0.0001$ & $1.398(1.176-1.661)$ & 0.0001 \\
\hline Previous prostate biopsy history & $0.949(0.592-1.519)$ & 0.8260 & $0.962(0.591-1.565)$ & 0.8758 \\
\hline \multicolumn{5}{|l|}{ Cancer } \\
\hline Age (yr) & $1.070(1.047-1.093)$ & $<0.0001$ & $1.069(1.043-1.096)$ & $<0.0001$ \\
\hline PSA (ng/mL) & $1.064(1.032-1.097)$ & $<0.0001$ & - & - \\
\hline Prostate volume $(\mathrm{mL})$ & $0.972(0.962-0.982)$ & $<0.0001$ & - & - \\
\hline PSA density ( 0.1 unit) & $1.683(1.437-1.972)$ & $<0.0001$ & $1.625(1.372-1.925)$ & $<0.0001$ \\
\hline Previous prostate biopsy history & $0.616(0.415-0.914)$ & 0.0161 & $0.538(0.339-0.853)$ & 0.0084 \\
\hline Presence of visible lesions & $7.212(4.566-11.389)$ & $<0.0001$ & $5.305(3.244-8.674)$ & $<0.0001$ \\
\hline \multicolumn{5}{|l|}{ Significant prostate cancer } \\
\hline Age (yr) & $1.072(1.049-1.096)$ & $<0.0001$ & $1.072(1.045-1.100)$ & $<0.0001$ \\
\hline PSA $(\mathrm{ng} / \mathrm{mL})$ & $1.083(1.050-1.117)$ & $<0.0001$ & - & - \\
\hline Prostate volume $(\mathrm{mL})$ & $0.974(0.964-0.985)$ & $<0.0001$ & - & - \\
\hline PSA density ( 0.1 unit) & $1.691(1.466-1.951)$ & $<0.0001$ & $1.632(1.405-1.894)$ & $<0.0001$ \\
\hline Previous prostate biopsy history & $0.746(0.502-1.110)$ & 0.1480 & $0.730(0.457-1.165)$ & 0.1869 \\
\hline Presence of visible lesions & $10.327(5.746-18.563)$ & $<0.0001$ & $7.413(3.965-13.861)$ & $<0.0001$ \\
\hline
\end{tabular}

PSA: prostate-specific antigen, CI: confidence interval.

cutoff values calculated from the Youden index was determined. A PSA density of $0.15 \mathrm{ng} / \mathrm{mL} / \mathrm{cm}^{3}$ was identified as the optimal cutoff point, at which sensitivity is higher than 0.7 and the specificity is maximized, for predicting the presence of visible lesions on MRI as well as cancer diagnosis and 
Table 3. Diagnostic performance of prostate-specific antigen density for the presence of visible lesions, cancer, and clinically significant prostate cancer

\begin{tabular}{lccc}
\multicolumn{1}{c}{ Outcome } & Optimal cutoff point & Sensitivity $(95 \%$ CI $)$ & Specificity $(95 \%$ CI $)$ \\
\hline Presence of visible lesion & $\geq 0.15$ & $0.695(0.652-0.737)$ & $0.520(0.432-0.608)$ \\
Cancer & $\geq 0.15$ & $0.772(0.727-0.816)$ & $0.534(0.470-0.599)$ \\
Clinically significant cancer & $\geq 0.15$ & $0.806(0.759-0.853)$ & $0.490(0.434-0.546)$ \\
\hline
\end{tabular}

CI: confidence interval.

csPCa (Table 3).

\section{Usefulness of PSA Density in Evaluating the Efficacy of Prebiopsy MRI}

A subanalysis was performed for evaluating the efficacy of target biopsy in increasing prostate cancer detection rates compared with that of systematic biopsy. Among the 561 lesions detected, 27 (8.2\%) were diagnosed as prostate cancer concomitant with negative systematic biopsy results. In contrast, 60 lesions (26.1\%) were diagnosed as prostate cancer using systematic biopsy concomitant with negative target biopsy results.

In the groups with negative systematic biopsy results, a PSA density of $0.15 \mathrm{ng} / \mathrm{mL} / \mathrm{cm}^{3}$ was identified as the significant cutoff value for predicting a positive target biopsy $(\mathrm{p}=0.0019)$.

\section{DISCUSSION}

Age, PSA, prostate volume, PSA density, and previous biopsy history are well-known variables related to prostate cancer diagnosis. ${ }^{10}$ In the ROC curve analysis for the prediction of visible lesions on MRI, prostate cancer, and csPCa, the AUC of PSA density showed superior probability than those of PSA level and prostate volume. Therefore, we chose PSA density for the analysis to avoid confounding factors. Interestingly, the cutoff value of $0.15 \mathrm{ng} / \mathrm{mL} / \mathrm{cm}^{3}$ for PSA density was significant for predicting the presence of visible lesions, cancer diagnosis, and csPCa. The presence of visible lesions has also been shown as related to the higher possibility of prostate cancer diagnosis, especially csPCa, consistent with the results of another study. ${ }^{6}$ Furthermore, an increasing PIRADS score of the visible lesions was positively related to PSA density. Therefore, we used the variables combining PSA density and presence of visible lesions for predicting the outcomes of the target lesions, instead of the PI-RADS score.

According to the American Urological Association and Society of Abdominal Radiology, at least 2 targeted cores should be obtained from each MRIdefined target lesion. ${ }^{11}$ However, Lu et al. ${ }^{12}$ revealed that sampling of 2 cores of the target lesion misses nearly one-quarter of csPCa detected on additional sampling. To improve grade group prediction and limit upgrading risk, a recent study showed that at least 4 targeted biopsy cores should be considered. ${ }^{13}$ In this study, 4 MRI-targeted cores per target were performed. Owing to the invasiveness of a biopsy procedure, further study will be needed to decide the optimal number of targeted biopsy cores.

In the PAIREDCAP (Prospective Assessment of Image Registration in the Diagnosis of Prostate Cancer) study that compared targeted and systematic prostate biopsies for biopsy-naïve male patients, prostate cancer was detected up to $15 \%$ using systematic biopsy in the group without visible lesions, indicating a false-negative MRI result. ${ }^{14}$ Moreover, Bryant et al. ${ }^{15}$ reported that $15.1 \%$ of prostate cancer cases with a Gleason score $\geq 7(3+4)$ and with negative multiparametric MRI (mpMRI) findings were underdetected with systematic biopsy. In our study, $24.0 \%$ of the 
patients without visible lesions under mpMRI were diagnosed with prostate cancer, and approximately $11.2 \%$ had csPCa with a Gleason score $\geq 7(3+4)$. Generally, Asians have lower prostate volumes than Westerners; therefore, the PSA density is relatively high among Asians, resulting in higher cancer detection rates. In a previous study of 177 prostatectomy patients with nonvisible lesions, a nonvisible lesion was not a predictor for low-risk prostate cancer. ${ }^{16}$ Therefore, these findings indicate that a negative mpMRI result cannot rule out the nonperformance of prostate biopsy.

The PI-RADS v2 score was developed to assess csPCa, and the use of MRI for detecting prostate cancer has increased with the emergence of the PI-RADS. ${ }^{17}$ Mehralivand et al. ${ }^{18}$ reported that the cancer detection rates of $1,2,3,4$, and 5 categories in PI-RADS were 25.0\%, 20.2\%, 24.8\%, $39.1 \%$, and $86.9 \%$ for all prostate cancers and $0 \%$, 9.6\%, 12\%, 22.1\%, and $72.4 \%$ for csPCa, respectively. Our institution interpreted the presence of visible lesions on MRI as lesions with a PI-RADS score $\geq$ 3 (clinically significant cancer is equivocal). In our study, the PI-RADS score 3 vs. 4 vs. 5 for prostate cancer and csPCa were $30.9 \%$ vs. $61.2 \%$ vs. $90.1 \%$ and $14.9 \%$ vs. $44.9 \%$ vs. $83.7 \%$, respectively, which is similar to previous studies' results.

For MRI-target biopsy, a systematic biopsy in addition to target biopsy is recommended to achieve the best detection results. Targeted biopsy or systematic biopsy alone does not cover the overall detection rates. In a recent study, the overall cancer detection rates and those of target and systematic biopsies were $70 \%, 60 \%$, and 60\%, respectively, ${ }^{14}$ and we had comparable results in the present study, at 59.6\%, 59.0\%, and 55.1\%, respectively.

MRI yields a 5\%-16\% additional detection rate compared with systematic biopsy alone. Fourcade et al. reported that systematic biopsy alone would have missed the detection of prostate cancer in $12 \%$ of patients. ${ }^{19}$ In our study, 27 of 331 positive lesions (8.2\%) would have been missed if the targeted biopsy was not performed because of the false-negative MRI findings. In contrast, 60 of the negative lesions (26.1\%) would not have been diagnosed by targeted biopsy alone, which is similar to results of a previous study. ${ }^{20}$ Therefore, to maximize cancer detection rates, both targeted and systematic biopsies should be implemented. The present study also reported that the PSA density of $0.15 \mathrm{ng} / \mathrm{mL} / \mathrm{cm}^{3}$ was a useful indicator for recommending MRI to patients suspected with prostate cancer to prevent missed diagnoses in cases without targeted biopsy (Table 4).

Patients with prior negative biopsy results receiving a PI-RADS assessment category of 3 to 5 require a repeat biopsy with image guided targeting. ${ }^{11}$ In this setting, Exterkate et al. ${ }^{21}$ reported that the value of adding systematic biopsy to targeted biopsy is limited because only $1.3 \%$ csPCa would be missed if systematic biopsy had been omitted. In this study, however, 8\% csPCa would be missed without systematic biopsy. Therefore, we cannot confirm that repeat biopsy

Table 4. Stratifying outcomes of the target and systematic biopsies according to prostate-specific antigen (PSA) density

\begin{tabular}{lccc}
\hline \multicolumn{1}{c}{ Variable } & Target biopsy benign & Target biopsy cancer & p-value \\
\hline Systematic biopsy & & & $<0.0001$ \\
Benign & $170(73.91)$ & $27(8.16)$ & $304(91.84)$ \\
Cancer & $60(26.09)$ & & 0.0019 \\
Systematic biopsy (benign) & & $5(18.52)$ & \\
PSA density $<0.15 \mathrm{ng} / \mathrm{mL} / \mathrm{cm}^{3}$ & $86(50.59)$ & $22(81.48)$ & \\
PSA density $\geq 0.15 \mathrm{ng} / \mathrm{mL} / \mathrm{cm}^{3}$ & $84(49.41)$ & & \\
\hline
\end{tabular}


could be safely omitted despite prior biopsy negative history.

Washino et al. ${ }^{22}$ reported that a PI-RADS v2 score $\leq 3$ and PSA density $<0.15 \mathrm{ng} / \mathrm{mL} / \mathrm{cm}^{3}$ does not yield csPCa. Oishi et al. ${ }^{6}$ suggested that repeat biopsy could be omitted in cases with a negative MRI finding and those with a PSA density $<0.15 \mathrm{ng} / \mathrm{mL} / \mathrm{cm}^{3}$. As in previous studies, this study reconfirmed that the PSA density has a potential role in compensating for the low negative predictive value of mpMRI for prostate cancer. In other words, a PSA density $\geq 0.15$ $\mathrm{ng} / \mathrm{mL} / \mathrm{cm}^{3}$ is a useful indicator for detecting prostate cancer in target lesions concomitant with negative systematic biopsy results. Therefore, we recommend that patients with a PSA density $\geq 0.15$ $\mathrm{ng} / \mathrm{mL} / \mathrm{cm}^{3}$ should consider prebiopsy MRI.

Nevertheless, this study has some limitations. Several characteristics could account for the heterogeneity in the results, including the small cohort size, having multiple physicians in the study, and the variability of the clinical decisionmaking regarding performance of prebiopsy MRI. To increase the cost-effectiveness of prebiopsy MRI, the indicators to reduce false-positive MRI findings should be investigated. Despite these limitations, we demonstrated the indication for preventing missed prostate cancer diagnosis in cases without prebiopsy MRI.

\section{CONCLUSIONS}

A PSA density $\geq 0.15 \mathrm{ng} / \mathrm{mL} / \mathrm{cm}^{3}$ is a useful indicator for recommending prebiopsy MRI and for predicting cancer and csPCa. Therefore, patients with a PSA density $\geq 0.15 \mathrm{ng} / \mathrm{mL} / \mathrm{cm}^{3}$ should consider MRI to avoid a missed cancer diagnosis.

\section{CONFLICT OF INTEREST}

The authors claim no conflicts of interest.

\section{ORCID}

Jin Hyung Jeon, https://orcid.org/0000-0001-8663-1310

Kyo Chul Koo, https://orcid.org/0000-0001-7303-6256

Byung Ha Chung, https://orcid.org/0000-0001-9817-3660

Kwang Suk Lee, https://orcid.org/0000-0002-7961-8393

\section{REFERENCES}

1. Carroll PH, Mohler JL. NCCN Guidelines updates: prostate cancer and prostate cancer early detection. J Natl Compr Canc Netw 2018;16:620-3.

2. Schoots IG, Roobol MJ, Nieboer D, Bangma CH, Steyerberg EW, Hunink MG. Magnetic resonance imaging-targeted biopsy may enhance the diagnostic accuracy of significant prostate cancer detection compared to standard transrectal ultrasoundguided biopsy: a systematic review and metaanalysis. Eur Urol 2015;68:438-50.

3. Presti JC Jr, O'Dowd GJ, Miller MC, Mattu R, Veltri RW. Extended peripheral zone biopsy schemes increase cancer detection rates and minimize variance in prostate specific antigen and age related cancer rates: results of a community multi-practice study. J Urol 2003;169:125-9.

4. Lawrentschuk N, Haider MA, Daljeet N, Evans A, Toi A, Finelli A, et al. 'Prostatic evasive anterior tumours': the role of magnetic resonance imaging. BJU Int 2010;105:1231-6.

5. Ahmed HU, El-Shater Bosaily A, Brown LC, Gabe R, Kaplan R, Parmar MK, et al. Diagnostic accuracy of multi-parametric MRI and TRUS biopsy in prostate cancer (PROMIS): a paired validating confirmatory study. Lancet 2017;389:815-22.

6. Oishi M, Shin T, Ohe C, Nassiri N, Palmer SL, Aron $\mathrm{M}$, et al. Which patients with negative magnetic resonance imaging can safely avoid biopsy for 
prostate cancer? J Urol 2019;201:268-76.

7. Hansen NL, Barrett T, Kesch C, Pepdjonovic L, Bonekamp D, O'Sullivan R, et al. Multicentre evaluation of magnetic resonance imaging supported transperineal prostate biopsy in biopsy-naive men with suspicion of prostate cancer. BJU Int 2018; 122:40-9.

8. Weinreb JC, Barentsz JO, Choyke PL, Cornud F, Haider MA, Macura KJ, et al. PI-RADS Prostate Imaging - reporting and data system: 2015, version 2 . Eur Urol 2016;69:16-40.

9. Unal I. Defining as optimal cut-point value in ROC analysis: an alternative approach. Comput Math Methods Med 2017;2017:3762651.

10. Lsabel H. PSA screening-a matter of debate? Memo 2019;12;244-8.

11. Rosenkrantz AB, Verma S, Choyke P, Eberhardt SC, Eggener SE, Gaitonde K, et al. Prostate magnetic resonance imaging and magnetic resonance imaging targeted biopsy in patients with a prior negative biopsy: a consensus statement by AUA and SAR. J Urol 2016;196:1613-8.

12. Lu AJ, Syed JS, Ghabili K, Hsiang WR, Nguyen KA, Leapman MS, et al. Role of core number and location in targeted magnetic resonance imaging-ultrasound fusion prostate biopsy. Eur Urol 2019;76:147.

13. Ploussard G, Beauval JB, Renard-Penna R, Lesord M, Manceau C, Almeras C, et al. Assessment of the minimal targeted biopsy core number per MRI lesion for Improving prostate cancer grading prediction. J Clin Med 2020;9:225.

14. Elkhoury FF, Felker ER, Kwan L, Sisk AE, Delfin M, Natarajan S, et al. Comparison of targeted vs systematic prostate biopsy in men who are biopsy naive: the Prospective Assessment of Image Registration in the Diagnosis of Prostate Cancer (PAIREDCAP) study. JAMA Surg 2019;154:811-8.

15. Bryant RJ, Hobbs CP, Eyre KS, Davies LC, Sullivan ME, Shields W, et al. Comparison of prostate biopsy with or without prebiopsy multiparametric magnetic resonance imaging for prostate cancer detection: an observational cohort study. J Urol 2019; 201:510-19.

16. Lee SH, Koo KC, Lee DH, Chung BH. Nonvisible tumors on multiparametric magnetic resonance imaging does not predict low-risk prostate cancer. Prostate Int 2015;3:127-31.

17. Purysko AS, Rosenkrantz AB, Barentsz JO, Weinreb JC, Macura KJ. PI-RADS version 2: a pictorial update. Radiographics 2016;36:1354-72.

18. Mehralivand S, Bednarova S, Shih JH, Mertan FV, Gaur S, Merino MJ, et al. Prospective evaluation of PI-RADSTM version 2 using the International Society of Urological Pathology prostate cancer grade group system. J Urol 2017;198:583-90.

19. Fourcade A, Payrard C, Tissot V, Perrouin-Verbe MA, Demany N, Serey-Effeil S, et al. The combination of targeted and systematic prostate biopsies is the best protocol for the detection of clinically significant prostate cancer. Scand J Urol 2018;52:1749.

20. Freifeld Y, Xi Y, Passoni N, Woldu S, Hornberger B, Goldberg K, et al. Optimal sampling scheme in men with abnormal multiparametric MRI undergoing MRI-TRUS fusion prostate biopsy. Urol Oncol 2019;37:57-62.

21. Exterkate L, Webelin O, Barentsz JO, van der Leest MG, Kumer JA, Vreuls W, et al. Is there still a need for repeated systematic biopsies in patients with previous negative biopsies in the era of magnetic resonance imaging-targeted biopsies of the prostate? Eur Urol Oncol 2020;3:216-23.

22. Washino S, Okochi T, Saito K, Konishi T, Hirai M, Kobayashi Y, et al. Combination of prostate imaging reporting and data system (PI-RADS) score and prostate-specific antigen (PSA) density predicts biopsy outcome in prostate biopsy naive patients. BJU Int 2017;119:225-33. 